\title{
UNIQUENESS OF TORSION FREE CONNECTION ON SOME INVARIANT STRUCTURES ON LIE GROUPS
}

\author{
BY
}

\author{
MICHEL NGUIFFO BOYOM AND GEORGES GIRAUD
}

\begin{abstract}
Let $\subseteq$ be a connected Lie group with Lie algebra $\mathfrak{g}$. Let $\operatorname{Int}(\mathfrak{g})$ be the group of inner automorphisms of $\mathrm{g}$. The group $\mathcal{G}$ is naturally equipped with Int $(A)$-reductions of the bundle of linear frames on $\mathcal{S}$. We investigate for what kind of Lie group the 0 -connection of $\mathrm{E}$. Cartan is the unique torsion free connection adapted to any of those Int $(g)$-reductions.
\end{abstract}

1. Definitions and main results. Let $M$ be an $n$-dimensional manifold and let $G$ be a Lie subgroup of the linear group $G l\left(\mathbf{R}^{n}\right)$, with Lie algebra (S). All manifolds we shall consider are smooth and connected. Let us consider a $G$-reduction $E(M, G)$ of the frame bundle $E^{0}\left(M, G l\left(\mathbf{R}^{n}\right)\right)$ and two linear connections $\nabla_{1}$ and $\nabla_{2}$ adapted to $E(M, G)$. Suppose these connections have the same torsion tensor, so that

$$
\left(\nabla_{1}\right)_{X} Y-\left(\nabla_{1}\right)_{Y} X-[X, Y]=\left(\nabla_{2}\right)_{X} Y-\left(\nabla_{2}\right)_{Y} X-[X, Y]
$$

or

$$
\left(\nabla_{1}-\nabla_{2}\right)_{X} Y-\left(\nabla_{1}-\nabla_{2}\right)_{Y} X=0
$$

for any vector fields $X, Y$ on $M$. Then if one identifies the tangent space $T_{x}(M)$ for $x \in M$, with $\mathbf{R}^{n}$, the difference $\nabla_{1}-\nabla_{2}$ appears as an element of the space $\mathbf{R}^{n^{*}} \otimes(B) \cap S^{2} \mathbf{R}^{n^{*}} \otimes \mathbf{R}^{n}$ which is known to be the first prolongation of (S) (see [4]).

A $G$-structure $E(M, G)$ is said to be 1-flat if it can be equipped with a torsion free linear connection. Thus any 1 -flat $G$-structure can be equipped with at most one torsion free linear connection if and only if the first prolongation of $\&$ is zero.

We are concerned with the following problem. Let $(M, \omega)$ be a differentiable manifold equipped with a torsion free linear connection $\omega$. We wish to describe those linear subgroups $G$ such that the connection $\omega$ is the unique linear connection adapted to some $G$-reduction of the frame bundle of $M$. Obviously a necessary condition is that the first prolongation of the holonomy algebra of $\omega$ be zero. So if $\mathcal{H}_{\omega}$ is the holonomy algebra of $\omega$, the problem of finding all linear Lie groups with the previous properties is equivalent to that of finding all Lie subalgebras $\mathbb{S}$ of $\operatorname{End}\left(\mathbf{R}^{n}\right)$ such that

$\left(\mathrm{p}_{1}\right)$

$\left(\mathrm{p}_{2}\right)$

$$
\begin{aligned}
& \mathcal{H}_{\omega} \subset \text { (S) } \\
& \mathcal{H S}^{(1)}=0 .
\end{aligned}
$$

Received by the editors October 19, 1981 and, in revised form, July 27, 1982 and January 3, 1983. 1980 Mathematics Subject Classification. Primary 53C10, 53C05; Secondary 22E60.

Key words and phrases. G-structure, torsion free connection, prolongation, symmetric operator of Lie algebra.

C1983 American Mathematical Society $0002-9947 / 83 \$ 1.00+\$ .25$ per page 
In what follows we deal with differentiable manifolds $(\mathcal{G}, \omega)$ which are Lie groups equipped with the 0 -connection of Cartan. Our interest in this particular case is due to the fact that the 0-connection of Cartan describes the local structure of the Lie group $\mathcal{G}$. In particular, the 0 -connection of Cartan is invariant by the left translations of $\mathcal{G}$, while if $\nabla$ is the covariant derivative associated to the 0 -connection then $\nabla_{X} Y=\frac{1}{2}[X, Y]$ for any two left invariant vector fields $X$ and $Y$ on $\mathcal{G}$.

As a direct consequence of the above facts one deduces that the holonomy algebra, $\mathcal{H}_{0}$, of the 0 -connection is included in the Lie algebra ad( $g$ ) of inner derivations of $g$ ( $g$ being the Lie algebra of $\mathcal{G}$ ). Let $\operatorname{Int}(g)$ be the connected Lie subgroup of $G l(g)$ associated to ad( $g$ ). Let us extend the holonomy fiber bundles of the 0 -connection to $\operatorname{Int}(g)$-reductions of the frame bundle of $\mathcal{G}$ to get left invariant Int $(g)$-structures. Any two such extensions are conjugate.

Our main results give a characterization of those Lie groups $\mathcal{G}$ on which the $\operatorname{Int}(g)$-structures constructed as above belong to the set of $\operatorname{Int}(g)$-reductions of the frame bundle of $\mathcal{G}$ which satisfy the properties $\left(\mathrm{p}_{1}\right)$ and $\left(\mathrm{p}_{2}\right)$, so that $\mathcal{H}_{0} \subset \operatorname{ad}(\mathfrak{g})$ and $(\operatorname{ad}(g))^{(1)}=0$. For such a Lie group $\mathcal{G}$, the 0 -connection of Cartan is the unique torsion free linear connection adapted to its holonomy bundles. We make technical use of a Lie subalgebra $\mathfrak{h}_{\mathfrak{g}}$ of the linear Lie algebra End $(g)$, which is defined as follows. A linear endomorphism $\varphi$ of the vector space $\mathfrak{g}$ belongs to $\mathfrak{h}_{\mathfrak{g}}$ if it satisfies the identity

$$
[\varphi(X), Y]+[X, \varphi(Y)]=0
$$

for any pair $(X, Y)$ in $\mathfrak{g} \times \mathfrak{g}$. Such a $\varphi$ is called a symmetric operator of $\mathfrak{g}$. In the present work we restrict ourselves to the case of nonsolvable Lie groups.

Now let us denote by $r$ the radical of the Lie algebra $g$, i.e., $r$ is the maximal solvable ideal in $\mathfrak{g}$. Taking a Levi subalgebra $\mathfrak{s}$ of $\mathfrak{g}$, the vector space $\mathfrak{g}$ becomes a direct sum: $\mathfrak{g}=\mathfrak{r} \oplus \mathfrak{g}$. Let us consider $\mathfrak{r}$ with its $\mathfrak{b}$-module structure given by the extension $0 \rightarrow \mathfrak{r} \rightarrow \mathfrak{g} \rightarrow \mathfrak{g} \rightarrow 0$. The subspace of $r$ consisting of $\mathfrak{g}$-invariant elements is denoted by $r^{\mathfrak{b}}$. As $\mathfrak{g}$ is a semisimple Lie algebra, the subspace $[\mathfrak{b}, \mathfrak{r}]$ is a submodule of the $\mathfrak{s}$-module $r$, and one gets the direct sum of $\mathfrak{s}$-modules

$$
r=r^{\mathfrak{s}} \oplus[\mathrm{r}, \mathfrak{s}] \text {. }
$$

The maximal ideal of $g$ contained in $r^{\mathfrak{s}}$ is denoted $D_{\mathfrak{g}}^{\infty}\left(\mathrm{r}^{\mathfrak{b}}\right)$ and the center of the subalgebra $r^{\mathfrak{B}}$ is denoted $Z\left(r^{\mathfrak{B}}\right)$. The subspace of $r^{\mathfrak{B}}$ denoted by $h_{r^{8}}\left(r^{\mathfrak{B}}\right)$ is that obtained by the evaluation map of $h_{r^{*}} \otimes r^{3}$ in $r^{3}$.

The main geometrical results to be proved are the following.

$\left(\mathcal{R}_{1}\right)$ Let $\mathcal{G}$ be a Lie group and let $\mathfrak{g}$ be its Lie algebra. Then the 0 -connection $\nabla_{0}$ of Cartan is the unique torsion free connection on each $\operatorname{Int}(g)$-extension of any holonomy bundle of $\nabla_{0}$ if and only if the ideal $h_{r^{8}}\left(r^{\mathfrak{s}}\right) \cap D_{\mathfrak{g}}^{\infty}\left(\mathrm{r}^{\mathfrak{5}}\right)$ is included in the center of $r^{b}$.

$\left(\Re_{2}\right)$ Let $\mathcal{G}$ be a Lie group and let $\mathfrak{g}$ be its Lie algebra. Suppose that $r^{\mathfrak{g}}$ is a commutative subalgebra of $g$. Then one gets uniqueness of torsion free connection adapted to each $\operatorname{Int}(g)$-extension of any holonomy bundle of $\nabla_{0}$ if and only if the Lie group $\mathcal{G}$ has discrete center. 
$\left(\mathscr{G}_{3}\right)$ Take $\mathcal{G}$ to be a Lie group, the radical of which is nilpotent subgroup. If $\mathcal{G}$ has discrete center, then there is a unique torsion free connection on each $\operatorname{Int}(\mathrm{g})$-extension of the holonomy bundle of $\nabla_{0}$.

$\left(\Re_{4}\right)$ Given a Lie group $\mathcal{G}$, let $\Re^{\mathfrak{S}}$ be the connected Lie subgroup of $\mathcal{G}$ associated to the Lie subalgebra $r^{\mathfrak{s}}$. If $R^{\mathfrak{s}}$ is a normal subgroup, then one gets uniqueness of the torsion free connection on $\operatorname{Int}(g)$-extension of the holonomy bundle of $\nabla_{0}$ if and only if the same result holds on the Lie group $R^{5}$.

2. Algebraic results. Because of the left invariant character of the previous results we shall deal with their infinitesimal versions. Thus, at the Lie algebra level we are concerned with finite-dimensional Lie algebras on a field $K$ of characteristic zero.

THEOREM 1. For any linear endomorphism $\varphi$ of $g$ which belongs to the Lie algebra $h_{\mathfrak{g}}$ the following assertions hold:

(i) The restriction of $\varphi$ to the subspace $[\mathrm{r}, \mathfrak{I}] \oplus \mathfrak{S}$ takes its values in the center of the Lie algebra g.

(ii) The restriction of $\varphi$ to the Lie algebra $\mathrm{r}^{\mathfrak{5}}$ is an element of the Lie algebra $h_{\mathrm{r}^{\mathfrak{5}}}$ and takes its values in the subspace $\mathrm{r}^{[\mathrm{r}, \mathrm{s}]}$ of elements in $\mathrm{r}$ which commute with the subspace $[r, \mathfrak{s}]$.

Before starting the proof of Theorem I, let us remark that our interest in the Lie algebra $h_{\mathrm{g}}$ arises from the following facts. Let $g$ be a Lie algebra and let $h_{\mathfrak{g}}^{0}$ be the vector space of all linear maps of $g$ into its center $Z(g)$. Consider the linear map $\pi$ of $h_{\mathrm{g}}$ into $(\operatorname{ad}(\mathrm{g}))^{(1)}$ given by $\pi(\varphi)=$ ad $\circ \varphi, \varphi \in h_{\mathfrak{g}}$, so that for any element $X$ in $\mathrm{g}$ one gets $\pi(\varphi)(X)=\operatorname{ad}_{\varphi(X)}$. It is clear that the bilinear map $(X, Y) \rightarrow[\varphi(X), Y]$ of $\mathfrak{g} \times \mathfrak{g}$ in $\mathfrak{g}$ is symmetric. Thus the previous map $\pi$ takes its values in the first prolongation of $\operatorname{ad}(g)$. This map is onto because of the definition of $(\operatorname{ad}(g))^{(1)}$. The kernel of $\pi$ is $h_{\mathfrak{g}}^{0}$. So one obtains the following exact sequence of vector spaces:

$$
0 \rightarrow h_{\mathrm{g}}^{0} \rightarrow h_{\mathrm{g}} \rightarrow(\operatorname{ad}(\mathrm{g}))^{(1)} \rightarrow 0 \text {. }
$$

Since the Cartan-Killing form $(X, Y) \mapsto \Phi(X, Y)=\operatorname{Tr}\left(\operatorname{ad}_{X} \circ \operatorname{ad}_{Y}\right)$ of $g$ is invariant by the inner derivations of $\mathfrak{g}$, for each $\varphi$ in $h_{\mathfrak{g}}$ one gets

$$
\begin{aligned}
\Phi([\varphi(X), Y], Z) & =-\Phi(Y,[\varphi(X), Z])=-\Phi(Y,[\varphi(Z), X]) \\
& =\Phi([\varphi(Z), Y], X)=\Phi([\varphi(Y), Z], X)=-\Phi(Z,[\varphi(Y), X]) \\
& =-\Phi([\varphi(Y), X], Z)=-\Phi([\varphi(X), Y], Z) .
\end{aligned}
$$

Thus $\Phi([\varphi(X), Y], Z)=\Phi(\varphi(X),[Y, Z])=0$, and the image $\varphi(g)$ is perpendicular to $[\mathfrak{g}, \mathfrak{g}]$ under $\Phi$. As is well known this implies that $\varphi(\mathfrak{g})$ lies in the radical $\mathfrak{r}$ of $\mathfrak{g}$.

For an element $\varphi$ in $h_{\mathrm{g}}$ let us denote by $A$ and $B$ the restriction of $\varphi$ to $\mathrm{r}$ and to $\mathfrak{Z}$, respectively. Let $(r, s)$ and $\left(r^{\prime}, s^{\prime}\right)$ be two elements in $g \approx r \times g$. With respect to above notation one gets

$$
\left[(A(r)+B(s), 0),\left(r^{\prime}, s^{\prime}\right)\right]=\left[\left(A\left(r^{\prime}\right)+B\left(s^{\prime}\right), 0\right),(r, s)\right] .
$$

This last identity gives rise to the system

$$
\left[A(r), r^{\prime}\right]=\left[A\left(r^{\prime}\right), r\right],
$$




$$
\begin{aligned}
& {\left[B(s), r^{\prime}\right]=\left[A\left(r^{\prime}\right), s\right],} \\
& {\left[B(s), s^{\prime}\right]=\left[B\left(s^{\prime}\right), s\right] .}
\end{aligned}
$$

To prove Theorem I, we need two technical lemmas.

LEMMA 1. Let $\mathrm{g}$ be a Lie algebra such that its Levi subalgebras $\mathfrak{g}$ are 3-dimensional, and let $\mathrm{g}=\mathrm{r} \oplus \mathfrak{g}$ be a Levi decomposition of $\mathrm{g}$. Suppose $\mathrm{r}$ is an irreducible $\mathfrak{g}$-module of dimension greater than one. Then for any element $\varphi$ of $h_{\mathfrak{a}}$ the restriction $B$ of $\mathfrak{g}$ to $\varphi$ is zero.

Proof. One can suppose the ground field is algebraically closed. (This is done without loss of generality.) Let $m+1$ be the dimension of the radical of $g$. Since $s$ is a 3-dimensional semisimple Lie algebra, we can choose a basis $(X, Y, H)$ in 5 such that

$$
[X, Y]=H, \quad[H, X]=2 X, \quad[H, Y]=-2 Y .
$$

Let $v$ be a primitive element of the 5 -module $\mathrm{r}$. Then the sequence $v_{0}=v$, $v_{1}=Y v, \ldots, v_{m}=Y^{m} v$ is a basis of the vector space $r$ which satisfies the system

$$
\begin{aligned}
H . v_{i}=(m-2 i) v_{i}, \quad i=0,1, \ldots, m, & \\
Y . v_{i}=v_{i+1}, \quad i=0,1, \ldots, m-1 \text { and } Y . v_{m} & =0, \\
X . v_{0}=0 & \text { and } X . v_{i}=(-m i+i(i-1)) v_{i-1}, \quad i=1, \ldots, m,
\end{aligned}
$$

where, for any $s \in \mathfrak{s}$ and $r \in \mathrm{r}$ we write $s . r$ for $[s, r]$. Now from the relations (3) and the system (4) one obtains

$$
Y \cdot B(H)=H . B(Y), \quad Y . B(X)=X . B(Y), \quad H \cdot B(X)=X . B(H) .
$$

If one writes these in terms of the basis $\left(v_{i}\right)$, one gets

$$
\begin{aligned}
\sum_{i=0}^{m} B_{i}(H) Y . v_{i} & =\sum_{i=0}^{m} B_{i}(Y) H . v_{i}, \\
\sum_{i=0}^{m} B_{i}(X) Y . v_{i} & =\sum_{i=0}^{m} B_{i}(Y) X . v_{i}, \\
\sum_{i=0}^{m} B_{i}(X) H . v_{i} & =\sum_{i=0}^{m} B_{i}(H) X . v_{i},
\end{aligned}
$$

the $v_{i}$-components in (6) for $i=0,1, \ldots, m$, we have the relations

$$
B_{0}(Y)=0, \quad B_{1}(Y)=0, \quad B_{m-1}(X)=0, \quad B_{m}(X)=0,
$$

and for $1 \leqslant i \leqslant m-1$,

$$
\begin{aligned}
B_{i-1}(H) & =(m-2 i) B_{i}(Y), \\
B_{i-1}(X) & =(i+1)(-m+i) B_{i+1}(Y), \\
(m-2 i) B_{i}(X) & =(i+1)(-m+i) B_{i+1}(Y) .
\end{aligned}
$$

The last three equalities give

$$
(i+2)(m-2 i)(-m+i+1) B_{i+2}(Y)=(i+1)(-m+i)(m-2 i-4) B_{i+2}(Y) .
$$


Therefore, we get either $B_{i+2}(Y)=0$ or

$$
(i+2)(m-2 i)(m-i-1)=(i+1)(m-i)(m-2 i-4) .
$$

The ultimate equality implies $m(m+2)=0$; that cannot hold because $m$ is positive. If $i$ is an integer such that $2<i+2<m$ one gets $B_{i+2}(Y)=0$. This proves that $B(Y)=0$ and we conclude that $B(X)=B(H)=0$. Now we show that we can drop the condition that $r$ is an irreducible $\mathfrak{S}$-module.

Lemma 2. Let $\mathrm{g}$ be a Lie algebra such that its Levi subalgebras $\mathfrak{\xi}$ are 3-dimensional and let $\mathrm{g}=\mathrm{r} \oplus \mathrm{g}$ be a Levi decomposition of $\mathrm{g}$. For any element $\varphi$ of $h_{\mathfrak{\mathrm { a }}}$ the restriction

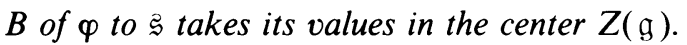

Proof. As in Lemma 1, let us suppose that the ground field is algebraically closed. Because of the simplicity of $\mathfrak{g}$, the radical $r$ is a direct sum of irreducible $\mathfrak{s}$-modules

$$
\mathrm{r}=I_{1} \oplus \cdots \oplus I_{t} \text {. }
$$

We know that if $\varphi$ is an element in $h_{\mathfrak{g}}$, the linear map $B$ of $s$ into $r$ which is deduced from $\varphi$ satisfies the relation $\left[B(s), s^{\prime}\right]=\left[B\left(s^{\prime}\right), s\right]$. Take $B_{I_{j}}$ to be the $I_{j}$-component of $B$. Then Lemma 1 tells us that, for any $I_{j}$ which has dimension greater than one, we get $B_{I_{1}}=0$, so that $B$ takes its values in the subalgebra $\mathfrak{r}^{\mathfrak{s}}$. The relation $[B(s), r]=[A(r), s]$ implies that $[B(s), r]$ lies in the $\mathfrak{s}$-module $[\mathfrak{s}, \mathrm{r}]$. The subspace $\mathrm{r}^{\mathfrak{5}}$ being a subalgebra of $\mathrm{g}$, the term $\left[B(s), r_{0}\right]$ also lies in $\mathrm{r}^{\mathfrak{5}}$ for any $\left(s, r_{0}\right)$ in $\mathfrak{s} \times \mathrm{r}^{\mathfrak{s}}$, so that we get $\left[B(s), r_{0}\right]=0$. Therefore, we see that $B$ takes its values in the center of $\mathrm{r}^{\mathfrak{s}}$. Thus if $s$ and $s^{\prime}$ are elements of $\mathfrak{g}$ and if $r \in \mathfrak{r}$ we get

$$
\left[s^{\prime},[B(s), r]\right]=\left[\left[s^{\prime}, B(s)\right], r\right]+\left[B(s),\left[s^{\prime}, r\right]\right]=\left[B(s),\left[s^{\prime}, r\right]\right],
$$

so that the inner derivation $\operatorname{ad}_{B(s)}$ of $\mathrm{r}$ is compatible with the action of $\mathfrak{s}$. This means that $\operatorname{ad}_{B(s)}$ is a $\mathfrak{s}$-module morphism.

Suppose that $I_{j}$ is an irreducible factor of the decomposition (7) with $\operatorname{dim} I_{j}>1$. The classical Schur lemma tells us that either $\operatorname{ad}_{B(s)}\left(I_{j}\right)$ is $\{0\}$ or $\operatorname{ad}_{B(s) \mid I}$ is an isomorphism. In the latter case the subspace $J_{j}=\left[B(s), I_{j}\right]$ is an irreducible $\mathfrak{s}$-module which is not zero. According to the formula (2), one gets the following commutative diagram:

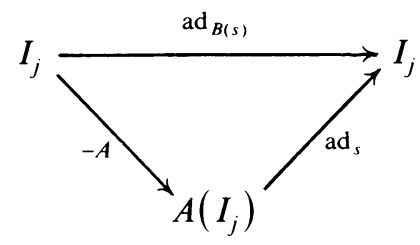

If $J_{j}$ is different from $\{0\}$ the above diagram implies that $A$ is an isomorphism of $I_{j}$ on $A\left(I_{j}\right)$ and idem for the restriction to $A\left(I_{j}\right)$ of $\mathrm{ad}_{s}$. We conclude that $A\left(I_{j}\right)$ is exactly the submodule $J_{j}$. Moreover, $J_{j}$ does not depend to the choice of $s$ in $\mathfrak{B}$. As we deal only with restrictions, the map $s \rightarrow \operatorname{ad}_{B(s)} \mid I_{j}$ is a linear map of $\mathfrak{s}$ in the space of

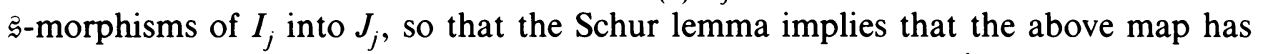
rank one. Finally, we deduce from the relation $-\operatorname{ad}_{B(s) \mid I_{j}} \circ A_{J_{j}}^{-1}=\operatorname{ad}_{s} \mid I_{j}$ that the 
kernel of $s \rightarrow \operatorname{ad}_{B(s)} \mid I_{j}$ is a nonzero ideal of $\mathfrak{s}$ different from $\mathfrak{s}$. The Lie algebra $\mathfrak{s}$ being simple, we get a contradiction.

Proof of Theorem I. Let us keep in mind that the ground field is algebraically closed. Let $C$ be a Cartan subalgebra of the Lie algebra $\mathfrak{3}$. Fix a simple system of roots $Q=\left(\alpha_{1}, \ldots, \alpha_{k}\right)$ associated to $C$. We write $\left(X_{i}, Y_{i}, H_{i}\right)$ for the Weyl system $\left(X_{\alpha_{i}}, Y_{\alpha_{i}}, H_{\alpha_{i}}\right.$ ) corresponding to the system $\mathcal{Q}$. As vector space, the Lie algebra $\mathfrak{s}$ is generated by the system $\left(X_{i}, Y_{i}, H_{i}\right), i=1,2, \ldots, k$. Let $\left(n_{i j}\right), i, j=1,2, \ldots, k$, be the Cartan matrix which is associated to $\mathcal{Q}$. For any $i=1,2, \ldots, k$, let $\mathfrak{S}_{i}$ be the Lie algebra $K X_{i} \oplus K Y_{i} \oplus K H_{i}$ (see [2, Chapter IV, §3]). Now let $\varphi$ be an element of $h_{\mathrm{g}}$. Lemma 2 guarantees that the vector subspace $\varphi\left(\mathfrak{g}_{i}\right)=B\left(\mathfrak{S}_{i}\right)$ is contained in the center of the subalgebra $\mathfrak{r} \oplus \mathfrak{s}_{i}$ of $\mathrm{g}$. If we consider the 2-cochain $X, Y \rightarrow-B[X, Y]$, then (3) is equivalent to the fact that the above 2-cochain is the coboundary of the 1-cochain $X \rightarrow B(X)$. Thus the 2-cochain $X, Y \rightarrow-B[X, Y]$ must be closed, so that

$$
[X, B[Y, Z]]-[Y, B[X, Z]]+[Z, B[X, Y]]=0
$$

for any $(X, Y, Z)$ in $\mathfrak{g} \times \mathfrak{g} \times \mathfrak{S}$. Now take $i, j$ in $[1,2, \ldots, k]$. According to the Weyl relations we may deduce from (8) that $\left[X_{i}, B\left[H_{i}, Y_{j}\right]\right]-\left[Y_{j}, B\left[H_{i}, X_{i}\right]\right]=$ $-n_{i j}\left[X_{i}, B\left(Y_{j}\right)\right]-2\left[Y_{j}, B\left(X_{i}\right)\right]=-\left(n_{i j}+2\right)\left[X_{i}, B\left(Y_{j}\right)\right]=0$. On the other hand, the relation (3) gives

$$
\left(n_{i j}+2\right)\left[X_{i}, B\left(Y_{j}\right)\right]=\left(n_{j i}+2\right)\left[X_{i}, B\left(Y_{i}\right)\right]=0 .
$$

For these last equalities to hold, the necessary condition is

$$
\left[X_{i}, B\left(Y_{j}\right)\right]=0 \text {. }
$$

Now let us compute the quantity $\left[H_{i}, B\left(Y_{j}\right)\right]$, taking

$$
\begin{aligned}
{\left[H_{i}, B\left(Y_{j}\right)\right] } & =\left[\left[X_{i}, Y_{i}\right], B\left(Y_{j}\right)\right]=\left[\left[X_{i}, B\left(Y_{j}\right)\right], Y_{i}\right]+\left[X_{i},\left[Y_{i}, B\left(Y_{j}\right)\right]\right] \\
& =\left[X_{i},\left[Y_{i}, B\left(Y_{j}\right)\right]\right]=\left[X_{i},\left[Y_{j}, B\left(Y_{i}\right)\right]\right] \\
& =\left[\left[X_{i}, Y_{j}\right], B\left(Y_{i}\right)\right]+\left[Y_{j},\left[X_{i}, B\left(Y_{i}\right)\right]\right] .
\end{aligned}
$$

If $i \neq j$, the Weyl relations together with Lemma 2 give

$$
\left[\left[X_{i}, Y_{j}\right], B\left(X_{i}\right)\right]=0 \text { and }\left[Y_{j},\left[X_{i}, B Y_{i}\right]\right]=0
$$

so that, for any $i, j$ in $[1,2, \ldots, k]$,

$$
\left[H_{i}, B\left(Y_{j}\right)\right]=0 \text {. }
$$

Finally, (9) and (10) tell us that for any $j=1,2, \ldots, k$ the element $\varphi\left(Y_{j}\right)=B\left(Y_{j}\right)$ (when it is not zero) is a primitive element in the $\mathfrak{g}$-module $r$ with the weight $0 \in C^{*}$. Therefore let us denote by $m_{j}$ the irreducible $\xi_{-}$-module generated by $\varphi\left(Y_{j}\right)$. It is well known that $m_{j}$ is generated as a vector space by the system $Y_{1}^{m_{1}} Y_{2}^{m_{2}} \cdots Y_{k}^{m_{k}} \cdot B\left(Y_{j}\right)$ where one identifies $Y_{i}$ with the operator $B\left(Y_{j}\right) \rightarrow\left[Y_{i}, B\left(Y_{j}\right)\right]$. On the other hand, 
$Y_{1}^{m_{1}} \cdots Y_{k}^{m_{h}} \cdot B\left(Y_{j}\right)$ has the weight $-\sum_{i=1}^{k} m_{i} \alpha_{i}$. In particular, let us compute the quantity $\left[H_{t},\left[Y_{i}, B Y_{j}\right]\right]$, taking

$$
\begin{aligned}
{\left[H_{t},\right.} & {\left.\left[Y_{i}, B\left(Y_{j}\right)\right]\right]=\left[\left[X_{t}, Y_{t}\right],\left[Y_{i}, B\left(Y_{j}\right)\right]\right] } \\
& =\left[X_{t},\left[Y_{t},\left[Y_{i}, B\left(Y_{j}\right)\right]\right]\right]-\left[Y_{t},\left[X_{t},\left[Y_{i}, B\left(Y_{j}\right)\right]\right]\right] \\
& =\left[X_{t},\left[Y_{t},\left[Y_{i}, B\left(Y_{j}\right)\right]\right]\right]=\left[X_{t},\left[\left[Y_{t}, Y_{i}\right], B\left(Y_{j}\right)\right]\right]+\left[X_{t},\left[Y_{i},\left[Y_{t}, B\left(Y_{j}\right)\right]\right]\right] \\
& =\left[X_{t},\left[\left[Y_{t}, Y_{i}\right], B\left(Y_{j}\right)\right]\right]=\left[X_{t},\left[Y_{j}, B\left(\left[Y_{t}, Y_{i}\right]\right)\right]\right]=\left[Y_{j},\left[X_{t}, B\left(\left[Y_{t}, Y_{i}\right]\right)\right]\right] \\
& =\left[Y_{j},\left[\left[Y_{t}, Y_{i}\right], B\left(X_{t}\right)\right]\right]=\left[Y_{j},\left[\left[Y_{t}, B\left(X_{t}\right)\right], Y_{i}\right]\right]+\left[Y_{j},\left[Y_{t},\left[Y_{i}, B\left(X_{t}\right)\right]\right]\right] \\
& =\left[Y_{j},\left[Y_{t},\left[Y_{i}, B\left(X_{t}\right)\right]\right]\right]=\left[Y_{j},\left[Y_{t},\left[X_{t}, B\left(Y_{i}\right)\right]\right]\right]=0 .
\end{aligned}
$$

This gives the identity

$$
\left[H_{t},\left[Y_{i}, B Y_{j}\right]\right]=0
$$

for any $i, j, t$ in $[1,2, \ldots, k]$. From the formulas (9) and (10) one gets $\left[X_{t},\left[Y_{i}, B\left(Y_{j}\right)\right]\right]$ $=0$ for any $i, j, t$ in $[1,2, \ldots, k]$. Thus (11) implies that $\left[Y_{i}, B\left(Y_{j}\right)\right]$ (if not zero) is a primitive element in $r$ with weight $0 \in K^{*}$. This contradicts the fact that any $Y_{i} B\left(Y_{j}\right)=\left[Y_{i}, B\left(Y_{j}\right)\right]$ is associated to the weight $-\alpha_{i}$. We see that $B$ takes its values in the center of the Lie algebra $g$, which proves part of (i). Let $A_{0}$ (resp. $A_{1}$ ) be the restriction to $\mathrm{r}^{\mathfrak{5}}$ (resp. to [r, $\left.\mathfrak{g}\right]$ ) of $\varphi \in h_{\mathrm{g}}$. It is a consequence of the exact sequence $0 \rightarrow h_{\mathfrak{g}}^{0} \rightarrow h_{\mathfrak{g}} \rightarrow \operatorname{ad}(\mathfrak{g})^{(1)} \rightarrow 0$ that the subspace $h_{\mathfrak{g}}(\mathfrak{g})$ generated by all the $\varphi(X)$, $\varphi \in h_{\mathfrak{g}}, X \in \mathfrak{g}$, is an ideal of the Lie algebra $\mathrm{g}$. In fact, take $(X, \varphi)$ in $\mathrm{g} \times h_{\mathfrak{g}}$ and define $X \varphi$ to be the element of End(g) defined by $Y \rightarrow(X \varphi)(Y)=[X, \varphi(Y)]-$ $\varphi[X, Y]$. One easily verifies that the map $X \varphi$ belongs to $h_{\beta}$, so that for any $X$ and $X^{\prime}$ in $g$ and for any $\varphi$ in $h_{\mathfrak{g}}$ the element $\left[X, \varphi\left(X^{\prime}\right)\right]$ lies in $h_{\mathfrak{g}}(g)$. Now take $r$ and $r^{\prime}$ in $r=r^{\mathfrak{s}}+[r, s]$. We may write

$$
r=r_{0}+r_{1}, \quad r^{\prime}=r_{0}^{\prime}+r_{1}^{\prime}
$$

where $r_{0}$ and $r_{0}^{\prime}$ (resp. $r_{1}$ and $r_{1}^{\prime}$ ) belong to $\mathrm{r}^{5}$ (resp. to [r, S]), to get

$$
\left[A_{0} r_{0}+A_{1} r_{1}, r_{0}^{\prime}+r_{1}^{\prime}\right]=\left[A_{0} r_{0}^{\prime}+A_{1} r_{1}^{\prime}, r_{0}+r_{1}\right] \text {. }
$$

This equation yields the three identities

$$
\begin{aligned}
& {\left[A_{1} r_{1}, r_{1}^{\prime}\right]=\left[A_{1} r_{1}^{\prime}, r_{1}\right],} \\
& {\left[A_{0} r_{0}, r_{0}^{\prime}\right]=\left[A_{0} r_{0}^{\prime}, r_{0}\right],} \\
& {\left[A_{0} r_{0}, r_{1}^{\prime}\right]=\left[A_{1} r_{1}^{\prime}, r_{0}\right] .}
\end{aligned}
$$

Given an element $s$ in $\mathfrak{s},(14)$ implies

$$
\left[s,\left[A_{1} r_{1}, r_{1}^{\prime}\right]\right]=\left[\left[s, A_{1} r_{1}\right], r_{1}^{\prime}\right]+\left[A_{1} r_{1},\left[s, r_{1}^{\prime}\right]\right] .
$$

Relation (2) together with Lemma 2 implies that the ideal $h_{\mathfrak{g}}(g)$ lies in the subalgebra $r^{5}$, so that we get

$$
\left[s,\left[A_{1} r_{1}, r_{1}^{\prime}\right]\right]=\left[A_{1} r_{1},\left[s, r_{1}^{\prime}\right]\right] .
$$


The first member $\left[s,\left[A_{1} r_{1}, r_{1}^{\prime}\right]\right]$ lies in the subspace $[\mathrm{r}, \mathfrak{g}]$, while the second member lies in the ideal $h_{\mathfrak{g}}(\mathfrak{g})$, so that $\left[A_{1} r_{1},\left[s, r_{1}^{\prime}\right]\right]=0$, and we obtain the equality

$$
\left[A_{1} r_{1},[\mathrm{r}, \mathfrak{s}]\right]=\{0\} \text {. }
$$

Bracketing $s \in \mathfrak{g}$ with both sides of (14) one gets

$$
\left[s,\left[A_{0} r_{0}, r_{1}^{\prime}\right]\right]=\left[A_{0} r_{0},\left[s, r_{1}^{\prime}\right]\right]=\left[s,\left[A_{1} r_{1}^{\prime}, r_{0}\right]\right]=0 .
$$

Our conclusion is

$$
\left[A_{0} r_{0},\left[s, r_{1}^{\prime}\right]\right]=\left[A_{1}\left[s, r_{1}^{\prime}\right], r_{0}\right]=0 .
$$

That ends the proof of (i). Proving (i), we established (13) and $\left[A_{0} r_{0},[\mathrm{r}, \mathfrak{g}]\right]=\{0\}$, so that (ii) holds and Theorem I is proved.

Applying Theorem I to a particular situation, we get the following

COROLlaRY I.1. Let $\mathrm{g}$ be a Lie algebra. Keeping the previous notations, suppose that the subalgebra $\mathrm{r}^{\mathfrak{5}}$ is commutative. Then the Lie algebra $\boldsymbol{t}_{\mathfrak{q}}$ is zero if and only if the center of $\mathfrak{g}$ is zero.

Proof. First, suppose that $h_{\mathfrak{g}}$ is zero. Then because of the inclusion of $h_{\mathfrak{g}}^{0}=Z(\mathfrak{g})$ $\otimes \mathfrak{g}^{*}$ in $h_{\mathfrak{g}}$ the center $Z(\mathfrak{g})$ of $\mathfrak{g}$ is zero. Second, suppose the center $Z(\mathfrak{g})$ of $\mathfrak{g}$ is zero. Let $\varphi$ be an element of $h_{\mathfrak{g}}$. For any element $r_{0}$ in $r^{\mathfrak{b}}$ the assertion (ii) of Theorem I tells us that the element $\varphi\left(r_{0}\right)$ commutes with the subspace $[\mathfrak{s}, r]$. Since $\mathfrak{r}^{\mathfrak{s}}$ is supposed to be commutative, $\varphi\left(r_{0}\right)$ lies in the center of $\mathfrak{g}$, which implies that the map $\varphi$ is identically zero.

EXAMPLE 2.1. Let $\mathcal{G}$ be any semisimple connected Lie group with Lie algebra $g$. Theorem I tells us that the Lie algebra $h_{\mathrm{g}}$ is zero, so that the exact sequence $0 \rightarrow h_{\mathfrak{g}}^{0} \rightarrow h_{\mathfrak{g}} \rightarrow(\operatorname{ad}(\mathfrak{g}))^{(1)} \rightarrow 0$ gives ad $(\mathfrak{g})^{(1)}=\{0\}$.

Keeping our previous notations, we have the following result.

THEOREM II. Let $\mathrm{g}$ be a Lie algebra and let us denote by $D_{\mathfrak{\beta}}^{\infty}\left(\mathrm{r}^{\mathfrak{s}}\right)$ the largest ideal of $\mathfrak{g}$ contained in $\mathrm{r}^{\mathfrak{s}}$. For any decomposition $\mathfrak{g}=\mathfrak{r} \oplus[\mathfrak{r}, \mathfrak{g}] \oplus \mathfrak{g}$ we have $h_{\mathfrak{g}}=$ $\operatorname{Hom}_{K}([\mathrm{r}, \mathfrak{g}] \oplus \mathfrak{g}, Z(\mathfrak{g})) \oplus\left(h_{\mathrm{r}^{\mathfrak{s}}} \cap \operatorname{Hom}_{K}\left(\mathrm{r}^{\mathfrak{s}}, D_{\mathfrak{g}}^{\infty}\left(\mathrm{r}^{\mathfrak{s}}\right)\right)\right)$.

Proof of Theorem II. Let us recall the construction of $D_{\mathfrak{g}}^{\infty}\left(\mathrm{r}^{\mathfrak{s}}\right)$ as it is given in [1]. We define the sequence $D_{\mathfrak{\beta}}^{i}\left(\mathrm{r}^{\mathfrak{s}}\right)$ by setting $D_{\mathfrak{g}}^{0}\left(\mathrm{r}^{\mathfrak{s}}\right)=\mathrm{r}^{\mathfrak{s}}$ and $D_{\mathfrak{g}}^{i+1}\left(\mathrm{r}^{\mathfrak{s}}\right)=$ $D^{1}\left(D_{\mathfrak{g}}^{i}\left(\mathrm{r}^{\mathfrak{s}}\right)\right)=\left\{X \in D_{\mathfrak{g}}^{i}\left(\mathrm{r}^{\mathfrak{b}}\right) /[X, \mathfrak{g}] \subset D_{\mathfrak{g}}^{i}\left(\mathrm{r}^{\mathfrak{j}}\right)\right\}, i \geqslant 0$. The ideal $D_{\mathfrak{g}}^{\infty}\left(\mathrm{r}^{\mathfrak{j}}\right)$ is the limit of the sequence $D_{\mathrm{g}}^{i}\left(\mathrm{r}^{\mathfrak{5}}\right)$.

First let us observe that $D_{\mathfrak{g}}^{\infty}\left(\mathrm{r}^{\mathfrak{s}}\right)$ is equal to $\mathrm{r}^{\mathfrak{s}} \cap \mathrm{r}^{[\mathrm{r}, \mathfrak{s}]}$. Indeed, if $\left(r_{0}, r_{1}, s\right)$ is an element of $\mathrm{r}^{\mathfrak{s}} \times \mathrm{r} \times \mathfrak{g}$, we have $\left[s,\left[r_{0}, r_{1}\right]\right]=\left[r_{0},\left[s, r_{1}\right]\right]$. The first member $\left[s,\left[r_{0}, r_{1}\right]\right]$ lies in the subspace $[r, \mathfrak{g}]$, so that we have the inclusion $\left[r^{\mathfrak{s}},[r, \mathfrak{g}]\right] \subset[r, \mathfrak{g}]$. Now, if $X$ is an element of $r^{\mathfrak{s}} \cap r^{[\mathfrak{r}, \mathfrak{s}]}$ and $\left(r_{0}, r_{1}\right) \in \mathfrak{r}^{\mathfrak{s}} \times[\mathfrak{r}, \mathfrak{g}]$, we get

$$
\left[\left[X, r_{0}\right], r_{1}\right]=\left[\left[X, r_{1}\right], r_{0}\right]+\left[X,\left[r_{0}, r_{1}\right]\right]=0 \text {. }
$$

We conclude that $\operatorname{ad}_{X}(\mathfrak{g})$ is included in $r^{\mathfrak{s}} \cap r^{[r, \tilde{s}]}$, so that $r^{\mathfrak{s}} \cap r^{[r, \mathfrak{s}]}$ is included in $D_{\mathfrak{g}}^{\infty}\left(\mathrm{r}^{\mathfrak{s}}\right)$. Conversely, let $(x, y, s)$ be an element of $D_{\mathfrak{g}}^{\infty}\left(\mathfrak{r}^{\mathfrak{g}}\right) \times \mathfrak{r} \times \mathfrak{g}$, so we have $[[x, y], s]-[x,[y, s]]=0$. The term $[[x, y], s]$ belongs to $[\mathrm{r}, \mathfrak{g}]$ while $[x,[y, s]]$ 
belongs to $[\mathfrak{r}, \mathfrak{s}]$, so that $\operatorname{ad}_{x}([\mathfrak{r}, \mathfrak{s}])=\{0\}$. Once we get $D_{\mathfrak{g}}^{\infty}\left(\mathfrak{r}^{\mathfrak{s}}\right)=\mathfrak{r}^{\mathfrak{s}} \cap \mathfrak{r}^{[\mathrm{r}, \mathfrak{s}]}$, Theorem I implies the inclusion

$$
h_{\mathfrak{g}} \subset \operatorname{Hom}_{K}([\mathrm{r}, \mathfrak{g}] \oplus \mathfrak{s}, Z(\mathfrak{g})) \oplus h_{\mathrm{r}^{\mathfrak{5}}} \cap \operatorname{Hom}_{K}\left(\mathrm{r}^{\mathfrak{s}}, D_{\mathfrak{g}}^{\infty}\left(\mathrm{r}^{\mathfrak{s}}\right)\right) .
$$

Conversely, any element $\left(\varphi_{0}, \varphi_{1}\right)$ of $\operatorname{Hom}_{K}([\mathrm{r}, \mathfrak{g}] \oplus \mathfrak{g}, Z(\mathfrak{g})) \oplus h_{\mathrm{r}^{5}} \cap$ $\operatorname{Hom}_{K}\left(\mathrm{r}^{\mathfrak{s}}, D_{\mathfrak{\beta}}^{\infty}\left(\mathrm{r}^{\mathfrak{s}}\right)\right)$ defines a unique element $\hat{\varphi}$ of $h_{\mathfrak{g}}$ by setting $\hat{\varphi}\left(r_{0}+r_{1}+s\right)=$ $\varphi_{0}\left(r_{1}+s\right)+\varphi_{1}\left(r_{0}\right)$. Indeed, according to the previous results we have

$$
\left[\varphi_{0}\left(r_{1}+s\right)+\varphi_{1}\left(r_{0}\right), r_{0}^{\prime}+r_{1}^{\prime}+s^{\prime}\right]=\left[\varphi_{1}\left(r_{0}\right), r_{0}^{\prime}\right]
$$

and

$$
\left[\varphi_{0}\left(r_{1}^{\prime}+s^{\prime}\right)+\varphi_{1}\left(r_{0}^{\prime}\right), r_{0}+r_{1}+s\right]=\left[\varphi_{1}\left(r_{0}^{\prime}\right), r_{0}\right]
$$

where $\left(r_{0}, r_{1}, s\right)$ and $\left(r_{0}^{\prime}, r_{1}^{\prime}, s^{\prime}\right)$ are elements of $\mathfrak{r}^{\mathfrak{s}} \times[\mathfrak{r}, \mathfrak{g}] \times \mathfrak{g} \simeq \mathfrak{g}$. Since $\varphi_{1}$ is an element of $h_{\mathrm{r}}$ : we have

$$
[\hat{\varphi}(X), Y]=[\hat{\varphi}(Y), X]
$$

for any pair $(X, Y)$ in $g \times \mathfrak{g}$. That proves the inclusion

$$
\operatorname{Hom}_{K}([\mathrm{r}, \mathfrak{g}] \oplus \mathfrak{g}, Z(\mathfrak{g})) \oplus h_{\mathrm{r}^{*}} \cap \operatorname{Hom}_{K}\left(\mathrm{r}^{\mathfrak{s}}, D_{\mathfrak{g}}^{\infty}\left(\mathrm{r}^{\mathfrak{s}}\right)\right) \subset h_{\mathfrak{g}}
$$

which ends the proof of Theorem II.

COROLlaRY II.1. For a Lie algebra $\mathfrak{g} \simeq \mathfrak{r}^{\mathfrak{s}} \oplus[\mathfrak{r}, \mathfrak{g}] \oplus \mathfrak{g}$, the space $h_{\mathfrak{g}}$ is zero if and only if the ideal $D_{\mathfrak{a}}^{\infty}\left(\mathrm{r}^{\mathfrak{5}}\right)$ is zero.

Proof. The sufficient condition is trivial. Conversely, let us suppose that $h_{\mathrm{g}}$ is zero. As we did before, we may suppose that the ground field $K$ is algebraically closed. If $D_{\mathfrak{a}}^{\infty}\left(\mathrm{r}^{\mathfrak{s}}\right)$ were different from zero, by applying a classical Lie theorem to the solvable Lie algebra $r$, one could find a nonzero element $v_{0}$ in $D_{\mathfrak{g}}^{\infty}\left(\mathfrak{r}^{\mathfrak{F}}\right)$ and a linear form $\lambda \in \mathrm{r}^{*}$ such that for any $X \in \mathfrak{r}$ one gets

$$
\left[X, v_{0}\right]=\lambda(X) v_{0} \text {. }
$$

Since $h_{\mathrm{g}}$ is zero, so is $Z(g)$, so that the linear form $\lambda$ is different from zero. Let us define the linear map $\varphi$ of $g$ into itself by putting

$$
\varphi(r+s)=\lambda(r) v_{0}
$$

for all $(r, s) \in \mathfrak{r} \times \mathfrak{g}$. Thus, given $(r, s)$ and $\left(r^{\prime}, s^{\prime}\right)$ in $\mathfrak{r} \times \mathfrak{g}$ we have

$$
\left[\varphi(r+s), r^{\prime}+s^{\prime}\right]=\left[\lambda(r) v_{0}, r^{\prime}+s^{\prime}\right]=\lambda(r)\left[v_{0}, r^{\prime}\right]=-\lambda(r) \lambda\left(r^{\prime}\right) v_{0}
$$

and

$$
\left[\varphi\left(r^{\prime}+s^{\prime}\right), r+s\right]=\left[\lambda\left(r^{\prime}\right) v_{0}, r+s\right]=\lambda\left(r^{\prime}\right)\left[v_{0}, r\right]=-\lambda\left(r^{\prime}\right) \lambda(r) v_{0} .
$$

We must conclude that the linear map $\varphi$ is a nonzero element of $h_{\mathrm{g}}$, which is contrary to our assumption. Corollary II.1 is proved.

COROLlaRY II.2. Let $\mathrm{g}$ be a Lie algebra with nilpotent radical $\mathrm{r}$. Then if the center $Z(g)$ is zero so is the Lie algebra $h_{g}$.

Proof. By Corollary II.1, if $h_{\mathfrak{g}}$ were not zero, the same would hold for the ideal $D_{\mathrm{g}}^{\infty}\left(\mathrm{r}^{\mathfrak{B}}\right)$. Applying the theorem of Engel, one would have a nonzero element $X_{0}$ in $D_{\mathfrak{g}}^{\infty}\left(\mathfrak{r}^{\mathfrak{g}}\right) \cap Z(\mathfrak{r})$. Such an element $X_{0}$ would lie in the center $Z(\mathfrak{g})$. 
Keeping in mind our geometrical interest in the prolongation $\operatorname{ad}(g)^{(1)}$, the previous results lead to this result.

THEOREM III. Let $\mathfrak{g}$ be a Lie algebra with a decomposition $\mathfrak{g} \simeq \mathfrak{r}^{\mathfrak{5}} \oplus[\mathrm{r}, \mathfrak{g}] \oplus \mathfrak{g}$. The first prolongation $(\mathrm{ad}(\mathrm{g}))^{(1)}$ of the linear space $\mathrm{ad}(\mathrm{g})$ is isomorphic to the factor space $h_{\mathrm{r}^{*}} \cap \operatorname{Hom}_{K}\left(\mathrm{r}^{\mathfrak{5}}, D_{\mathrm{q}}^{\infty}\left(\mathrm{r}^{\mathfrak{5}}\right)\right) / h_{\mathrm{r}^{*}} \cap \operatorname{Hom}_{K}\left(\mathrm{r}^{\mathfrak{5}}, Z(\mathrm{~g})\right)$.

The proof is an immediate consequence of Theorem II together with the exact sequence $0 \rightarrow \operatorname{Hom}(\mathfrak{g}, Z(\mathfrak{g})) \rightarrow h_{\mathfrak{g}} \rightarrow(\operatorname{ad}(\mathfrak{g}))^{(1)} \rightarrow 0$.

Corollary III.1. Let $\mathrm{g}$ be a Lie algebra with a Levi decomposition $\mathrm{r} \oplus \mathfrak{g}$. If $\mathrm{r}^{5}$ is commutative then $(\operatorname{ad}(\mathrm{g}))^{(1)}$ is zero.

Proof. We already proved that the ideal $D_{\alpha}^{\infty}\left(r^{\mathfrak{s}}\right)$ is equal to $r^{5} \cap r^{[r, \xi]}$. Since $r^{5}$ is commutative we get $h_{\mathrm{r}^{\mathrm{s}}}=\operatorname{Hom}\left(\mathrm{r}^{\mathfrak{s}}, \mathrm{r}^{\mathfrak{s}}\right)$ and $D_{\mathfrak{g}}^{\infty}\left(\mathrm{r}^{\mathfrak{s}}\right)=\mathrm{g}^{\mathrm{r}^{*}}=\mathrm{g}^{\mathfrak{g}}=Z(\mathrm{~g})$. Therefore, we have $h_{r^{*}} \cap \operatorname{Hom}\left(r^{\mathfrak{s}}, Z(\mathfrak{g})\right)=\operatorname{Hom}\left(r^{\mathfrak{s}}, Z(\mathfrak{g})\right)$.

Corollary III.2. Let $\mathrm{g}$ be a Lie algebra such that some $\mathrm{r}^{5}$ is an ideal in $\mathrm{g}$. Then $(\operatorname{ad}(\mathfrak{g}))^{(1)}$ is isomorphic to $\left(\operatorname{ad}\left(\mathrm{r}^{\mathfrak{5}}\right)\right)^{(1)}$.

Proof. Since $r^{\mathfrak{s}}$ is an ideal of $g$ we have $r^{\mathfrak{s}}=D_{\mathfrak{g}}^{\infty}\left(r^{\mathfrak{s}}\right)=r^{\mathfrak{s}} \cap r^{[r, \mathfrak{s}]}$. On the other hand, we can write $\left[r^{\mathfrak{5}}\right]^{r^{\mathfrak{s}}}=Z\left(r^{\mathfrak{5}}\right)$ so that

$$
Z\left(\mathrm{r}^{\mathfrak{s}}\right) \subset\left[\mathrm{r}^{\mathfrak{s}} \oplus[\mathrm{r}, \mathfrak{g}] \oplus \mathfrak{g}\right]^{\mathrm{r}^{*}}=Z(\mathfrak{g}) \text {. }
$$

That proves the equality $Z\left(\mathrm{r}^{5}\right)=Z(\mathfrak{g})$. We apply Theorem III and we obtain $(\operatorname{ad}(g))^{(1)} \simeq h_{r^{*}} / \operatorname{Hom}\left(r^{\mathfrak{5}}, Z\left(r^{5}\right)\right) \simeq\left(\operatorname{ad}\left(r^{\mathfrak{5}}\right)\right)^{(1)}$.

Proposition 2.1. Let $g$ be a Lie algebra. Then for any Levi subalgebra $\mathfrak{g}$, the subspace $h_{\mathrm{r}^{*}} \cap \operatorname{Hom}\left(\mathrm{r}^{\mathfrak{s}}, D_{\mathrm{g}}^{\infty}\left(\mathrm{r}^{\mathfrak{s}}\right)\right)$ is an ideal of $h_{\mathrm{r}}$. Furthermore, the subspace $h_{\mathrm{r}}\left(\mathrm{r}^{\mathfrak{s}}\right) \cap D_{\mathfrak{q}}^{\infty}\left(\mathrm{r}^{\mathfrak{S}}\right)$ is an ideal of $\mathrm{g}$ which does not depend on the choice of $\mathfrak{g}$.

Proof. Let $\varphi \in h_{\mathrm{r}^{*}}$ and let $\psi \in h_{\mathrm{r}^{*}} \cap \operatorname{Hom}\left(\mathrm{r}^{5}\right)$ so that $[\varphi, \psi]$ lies in $h_{\mathrm{r}^{*}}$. It remains to prove that for all $\left(r_{0}, r, s\right)$ in $\mathfrak{r}^{\mathfrak{s}} \times \mathrm{r} \times \mathfrak{s}$ we have $\left[[\varphi, \psi]\left(r_{0}\right),[r, s]\right]=0$. Here

$$
\begin{aligned}
{\left[[\varphi, \psi]\left(r_{0}\right),[r, s]\right] } & =\left[\varphi \psi\left(r_{0}\right)-\psi \varphi\left(r_{0}\right),[r, s]\right] \\
& =\left[\varphi \psi\left(r_{0}\right),[r, s]\right]=\left[\left[\varphi \psi\left(r_{0}\right), r\right], s\right] .
\end{aligned}
$$

If $i$ is an ideal of a Lie algebra $g$ and $\varphi \in h_{\mathfrak{g}}$, for all $v \in \mathfrak{g}$, we get $[\varphi(i), v]=$ $[\varphi(v), \mathfrak{i}] \subset \mathfrak{i}$ so that in the previous case $\left[\varphi \psi\left(r_{0}\right), r\right]$ lies in $D_{\mathfrak{g}}^{\infty}\left(\mathrm{r}^{\mathfrak{\xi}}\right)$ and the first statement holds. Now let $\mathfrak{g}_{1}$ and $\mathfrak{I}_{2}$ be two Levi subalgebras of $\mathfrak{g}$. A theorem of Malcev and Harish-Chandra tells us that there is an element $X_{0}$ of the nilpotent radical of $\mathfrak{g}$ such that $\mathfrak{S}_{2}=e^{\operatorname{ad}\left(X_{0}\right)}\left(\mathfrak{I}_{1}\right)$. Since $e^{\operatorname{ad}\left(X_{0}\right)}$ preserve every ideal of $\mathfrak{g}$ we have $e^{\operatorname{ad}\left(X_{0}\right)}\left(D_{\mathfrak{g}}^{\infty}\left(\mathrm{r}^{\xi_{1}}\right)\right)=D_{\mathfrak{g}}^{\infty}\left(\mathrm{r}^{\xi_{1}}\right)$. Since $\mathrm{r}^{\xi_{1}}$ and $\mathrm{r}^{\mathfrak{s}_{2}}$ must be conjugated by $e^{\operatorname{ad}\left(X_{0}\right)}$, so must the ideals $D_{\mathfrak{g}}^{\infty}\left(\mathrm{r}^{\mathfrak{s}_{1}}\right)$ and $D_{\mathfrak{g}}^{\infty}\left(\mathrm{r}^{\mathfrak{s}_{2}}\right)$, and one concludes that $D_{\mathfrak{g}}^{\infty}\left(\mathrm{r}^{\mathfrak{s}_{1}}\right)=D_{\mathfrak{g}}^{\infty}\left(\mathrm{r}^{\mathfrak{s}_{2}}\right)$.

Let us illustrate the main results by a few examples.

EXAMPLE 1. Let $g$ be a semisimple Lie algebra. The radical $r$ of $g$ being zero, Theorem I gives $h_{\mathfrak{g}}=\{0\}$, so that we get $(\operatorname{ad}(g))^{(1)}=\{0\}$. 
EXAMPLE 2. If $g$ is a reductive Lie algebra then we get $Z(g)=r^{\mathfrak{s}}=r$. By the Corollary III.1, we have $(\operatorname{ad}(\mathrm{g}))^{(1)}=\{0\}$.

EXAmple 3. Let $g$ be the affine Lie algebra $\mathbf{R}^{2} \times s l(2, \mathbf{R})$ and let $(u, X)$ be an element of $\mathrm{g}$. Then we get $\operatorname{ad}(u, X)=\left[\begin{array}{cc}X & -\delta u \\ 0 & \operatorname{ad} X\end{array}\right]$ where $\delta u(Y)=Y u$ for $Y \in s l(2, \mathbf{R})$. Since $\operatorname{sl}(2, \mathbf{R})$ is irreducible on $\mathbf{R}^{2}$, we have $r^{s /(2, \mathbf{R})}=\{0\}$, and Theorem III gives $(\operatorname{ad}(g))^{(1)}=\{0\}$.

EXAmple 4. Let $g$ be the Lie algebra $\mathbf{R}^{5} \# s l(2, \mathbf{R})$, with the bracket given by

$$
\begin{aligned}
& {\left[((a, b, c, \alpha, \beta), X),\left(\left(a^{\prime}, b^{\prime}, c^{\prime}, \alpha^{\prime}, \beta^{\prime}\right), X^{\prime}\right)\right]} \\
& \quad=\left(b c^{\prime}-b^{\prime} c+\alpha \beta^{\prime}-\alpha^{\prime} \beta, 0,0, X\left(\alpha^{\prime}, \beta^{\prime}\right)-X^{\prime}(\alpha, \beta),\left[X, X^{\prime}\right]\right) .
\end{aligned}
$$

Let us take $s$ to be the subalgebra $\{0,0,0,0,0\} \# s l(2, \mathbf{R})$. It is clear that

$$
\begin{aligned}
\mathrm{r}^{\mathfrak{s}} & =\mathbf{R}^{3} \times\{(0,0)\} \#\{0\}, \\
{[\mathrm{r}, \mathfrak{s}] } & =\{(0,0,0)\} \times \mathbf{R}^{2} \#\{0\}, \\
Z(\mathrm{~g}) & =Z\left(\mathrm{r}^{\mathfrak{5}}\right)=\mathbf{R} \times\{0,0,0,0\} \#\{0\} .
\end{aligned}
$$

Since $r^{\mathfrak{5}}$ is an ideal in $g$, by Corollary III.2, $(\operatorname{ad}(g))^{(1)}$ is isomorphic to the first prolongation of the inner derivations of the Heisenberg algebra $r^{5}$, which is the set of those $S \in \operatorname{Hom}\left(\mathrm{r}^{5} \times \mathrm{r}^{5}, \mathrm{r}^{5}\right)$ defined by

$$
S\left((a, b, c),\left(a^{\prime}, b^{\prime}, c^{\prime}\right)\right)=\left((\lambda b+\mu c) b^{\prime}+(\mu b+\nu c)\left(c^{\prime}, 0,0\right)\right.
$$

where $(\lambda, \mu, \nu) \in \mathbf{R}^{3}$.

3. Return to differential geometry. We begin by explaining the geometric interest of the ideal $\mathscr{G}=h_{\mathrm{r}^{\mathrm{s}}}\left(\mathrm{r}^{\mathfrak{5}}\right) \cap D_{\mathfrak{g}}^{\infty}\left(\mathrm{r}^{\mathfrak{5}}\right)$. One easily verifies that $\mathscr{G}$ is the minimal ideal of $\operatorname{ad}(g)$ such that the first prolongation of $\operatorname{ad}(g)$ coincides with that of $q$, so that

$$
(\operatorname{ad}(\mathfrak{g}))^{(1)}=g^{(1)} \text {. }
$$

This gives another understanding of Proposition 2.1. Moreover, the geometrical statement $\left(R_{1}\right)$ is a direct consequence of the above remark. The geometrical statements $\left(\Re_{2}\right),\left(\Re_{3}\right)$ and $\left(\Re_{4}\right)$ are consequences of Corollaries I.1, II.2 and III.2, respectively.

Take a left invariant torsion free connection $\nabla$ on a Lie group $\mathcal{G}$ and assume that its holonomy group is a subgroup of $\operatorname{Int}(g)$. One observes that the space $\operatorname{ad}(g)^{(1)}$ provides a parametrization of the set of all left invariant torsion free connections which are adapted to the $\operatorname{Int}(\mathrm{g})$-structure obtained from the holonomy bundle of $\nabla$ (see §1).

Our last remark applies to the case of solvable Lie groups which cannot be handled by the techniques used in this work. We may observe that for such a Lie group $\mathcal{G}$ with Lie algebra $g$ the linear Lie algebra $h_{\mathfrak{g}}$ is always different from zero. Let $g$ be a solvable Lie algebra. If $Z(g) \neq 0, \operatorname{Hom}_{K}(g, Z(g))$ is included in $h_{\mathfrak{g}}$. If $Z(g)=0$ then any $\xi$ in $Z([g, g])-\{0\}$ gives us a nonzero element $\operatorname{ad}_{\xi}$ in $h_{\mathfrak{g}}$. Thus, for any solvable Lie algebra with $Z(g)=0$ the first prolongation $\operatorname{ad}(g)^{(1)}$ is never zero.

ADDENDUM. The authors are indebted to the referee for many useful suggestions which led to better formulation of some theorems. 


\section{BIBLIOGRAPHY}

1. V. Guillemin, A Jordan Hölder decomposition for a certain class of infinite dimensional Lie algebras, J. Differential Geom. 2 (1968).

2. N. Jacobson, Lie algebras, Tracts in Math., no. 10, Interscience, New York, 1962.

3. J. Milnor, Curvatures of left invariant metrics on Lie groups, Adv. in Math. 21 (1976), 293-329.

4. I. Singer and S. Sternberg, The infinite groups of Lie and Cartan, J. Analyse Math. 15 (1965), $1-114$.

Universite des Sciences et Techniques du languedoc, Place Eugene Baraillon, 34060-MontPELlier CEDEX, France 